\title{
Improving presentation skill through gamified application - Gamification in practice
}

\author{
Eeva Leinonen*, Mika Haapaniemi *, Juho Mattila*, Aryan Firouzian*, Petri Pulli* \\ ${ }^{*}$ University of Oulu, ITEE Faculty, OASIS Group, Oulu, Finland \\ \{eeva.leinonen, aryan.firouzian, petri.pulli\}@oulu.fi \\ juho.mattila@ikunelabs.com \\ haapmika@gmail.com
}

\begin{abstract}
Gamification has been used for many years, as a major factor to enhance user's engagement in non-game context. This study focuses on design of an online peer support application to improve presentation skills among young unemployed users. Furthermore, the gamification principles such as pointing system and leaderboard panel are integrated into the design to encourage users for different activities. Remote test is conducted with 47 test subjects to investigate their attitudes toward gamification elements in the design. Pilot and assessment test are conducted with 5 and 7 test subjects to find usability flaws. The study results show significant user engagement after revealing the gamification elements in the system. Furthermore, the usability problems in the design are investigated.
\end{abstract}

\section{INTRODUCTION}

Based on Ministry of Economic Affairs and Employment report, there are total of 275600 job seekers, and 33400 under 25 years of age unemployed in Finland registered in the Employment and Economic Development Office. At the same time 17900 students attended the labor market training, and 40700 people have attended the self-motivated studies supported by unemployment benefits. [1]

In today's rapidly changing world, it is impossible to predict changes in the labor market, and keeping yourself in the game and promote your careers is even more challenging. People are increasingly expected to take responsibility to keep them labor-intensive. Some young people, who are currently graduating lack the skills to enter the labor market. Lifelong learning will be a key paradigm in training and education. Furthermore, information and communication technology is an important part to enable a wide range of learning tools and programs to help people and upgrade their skills easily, and guide their professional careers. [2]

There is lack of studies focusing on design and development of the applications to support job seeking skills on young employed people. Most of the research studies focus on the platform development of e-learning systems. Public vocational training can be seen as a resolved task to solve the unemployment problem for the modern government, while the e-learning technologies can be considered as a potential solution. Therefore, there is an essential need for research of user studies in gamified user-centered service design of usability, and user acceptance. For example, Ref . [3] has studied the unemployed vocational training research of acceptance of e-learning. Our study focused on designing the gamified application for improving presentation skill through usability and user friendly lens.

From the young job seekers point of view, the interview moment can be in challenges role in selection process [4], and often raises feelings of anxiety [5]. Furthermore, interview success can directly correlate with the opportunity to get jobs [6].

Games have motivated the humans in various activities since the dawn of time. Gamification, which Ref. [7] defines as "the use of game design elements in non-game contexts", plays a big role in modern application design as a driver for user-engagement.

Gamification is not game, but instead uses elements from game design in software design. Therefore, similar to games, gamification elements can engage users and encourage them to challenge themselves or others to improve their performance. [8]

One of the earliest and most successful applications that used game elements in its design was Foursquare. Since its release in 2009, the design principles that it introduced have been considered as foothold in interaction design and digital marketing [7]. Through gamification, game design has started to penetrate multiple areas of life, and especially in service design area [9].

It is crucial to have proper communications skills in business and in personal life. If you are a manager or a leader, you need to have the skills to get your message across people. [10]

A common way to implement design elements from games is setting achievements, which can be seen as goals in a reward system [9]. With the gamification definition presented earlier in mind, it could be said that in a gamified solution with achievements, users try to achieve a set of goals in order to get rewards or other forms of progress in non-game context.

It should be also noted that the video game achievement system has developed significantly during the last decade, by adding more challenges and play time to video games. Applications are made more engaging, hooking the user by the use of achievement systems. [11]

The design of user-engagement in EchoMe and its parent application HireMe was based on these gamification principles. 


\section{IMPLEMENTATION}

HireMe was designed as an application platform for mobile devices, aimed at unemployed young people, especially those who are in danger of isolation and dropping outside of working life.

The application platform was constructed to work as an ecosystem that brings the unemployed job-seekers and employers together through custom training games based on gamification design principles.

These custom games help the users to train their skills from which they gain experience points and achievements onto their personal user profiles. Then, this allows company recruiters follow the progress of the applicants and make comparisons between them, before continuing with the hiring process.

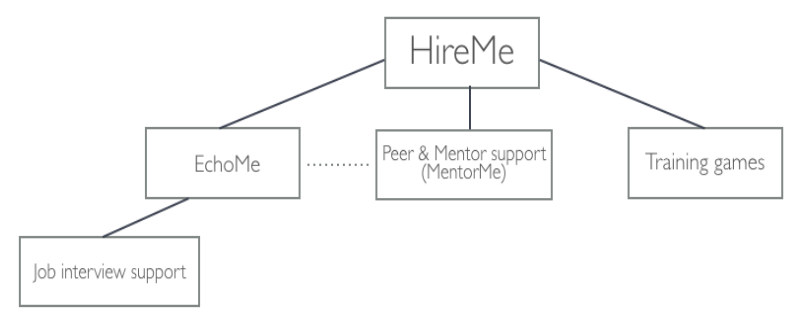

Figure 1. The ecosystem of HireMe platform

EchoMe, which this paper is concentrating on, is also designed to work with the platform in that its usage is motivated through a collection of points by using the application, which would be synced to the user account in HireMe.

\section{A. First prototype}

EchoMe - originally named EchoLive - was developed as a presentation skill improvement application. It is an online solution to improve the presentation skill through peer coaching. Elements of gamification principles are integrated later in the application to persuade the users to get involved in different scenarios.

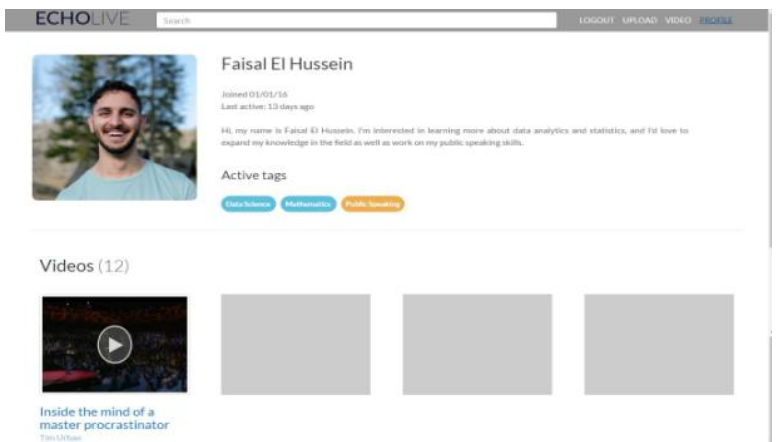

Figure 2. User profile page of the first EchoLive prototype implementation provides features to create and categorize video presentations, and start discussion on a video track, which appear when playing the video file. These features can already be seen in other web application such as SoundCloud, which is an online audio distribution platform.

Laravel and PHP framework was used to implement the database into the system. CSS, HTML and JavaScript were used for frontend user interface design. AngularJS was used for the real-time commenting system. The benefit of JavaScript is flexibility in implementation structure, which allows the developer to update and maintain the system with ease.

\section{B. Second prototype}

The second version - dubbed as EchoMe - brought the gamification elements it needed into play, as well as integration with the HireMe platform. All the points collected in EchoMe can be transferred onto HireMe by using the integrated accounts.

While all EchoLive functionalities are preserved, users can earn points by uploading videos and leaving comments. There is also a rating system to show quality of presentations and usefulness of comments. Finally, there is a leaderboard panel to sum points and rank first 100 users.

EchoMe included the following features:

- Logging in and creating a profile

- Searching for videos with search criteria

- Categorizing their uploaded videos

- Subscribing to other users to follow their content

- Uploading or streaming videos

- Tagging contributors, i.e. other users that participated in the video

- Commenting on videos in real time or go back to a point in the video and post a comment

- Up-voting and down-voting comments to indicate relevance and value

- Point system for users, where the net amount of comment up-votes and down-votes indicates their skill in criticism

- Point system for uploading, commenting and

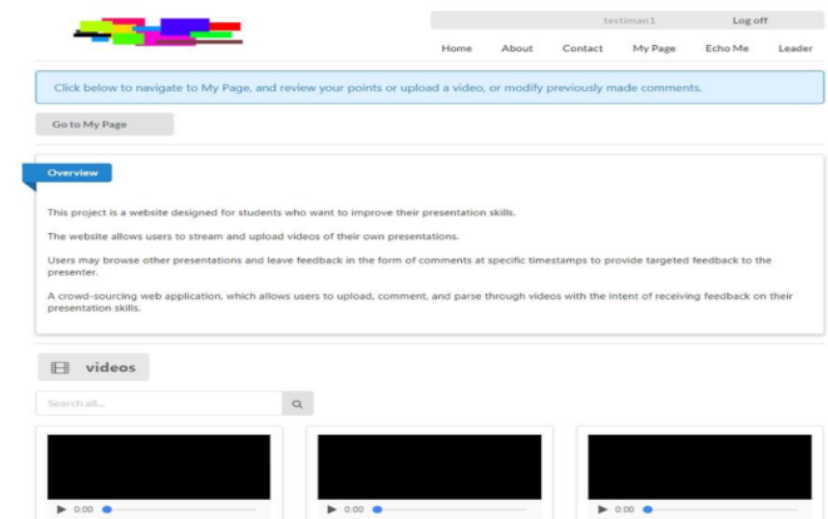

Figure 3. The EchoMe main screen
Since, EchoLive was developed as an online peer support tool to improve presentation skills, the EchoLive 
watching videos, which can be used by mentors to follow the activities of users.

The first EchoLive version does not support gamification elements in the design, and it derived us to develop EchoMe as the second prototype. It was designed through HTML language, jQuery library and MVC.Net framework. In Figure 3, you can see EchoMe page after you have logged in and clicked EchoMe tab. Then, the user can upload a video or search others' videos to comment and analyze them.

\section{EVALUATION}

The evaluation is conducted in three phases including remote, pilot and assessment usability tests. The remote usability test targets the idea of implementing gamification principles in the application to encourage users for different activities. The pilot and assessment usability tests are designed to evaluate the application to find usability flaws.

\section{A. Remote usability test}

EchoMe is used as an educational tool in IT Infrastructure course in Oulu University in 2017. About 50 students used EchoMe during the course, which lasted one and half months. Students chose a course related topic and recorded their video presentation of that topic. Then, they uploaded the video of their presentation into EchoMe and commented other presentations. Students were asked to upload an improved presentation video in the later-half of the course, but it was not a mandatory task. Users receive points for uploading, watching, commenting, rating and giving thumb-up on videos and comments. Then, sum of the points can be calculated based on those activities and received ratings and thumbs-ups. Value of the activity points can be setup in management panel, and in course scenario it is based on following scale:

- Uploading a video equals to 500 points

- Leaving a comment equals to 10 points

- Receiving thumb up equals to 5 points

- Watching a video equals to 1 point

- Average rate of the video is calculated through multiplying 1000 points by the rating grade out of five.

To investigate behavior change of the students in the presence of gamification elements, leaderboard and point system were not revealed to the students at the beginning of the course. They are revealed after first 20 days of using EchoMe. Then, user's activities were monitored in the next 20 days, seeking significant encouragement to get involved in the system.

Demographic data demonstrates that $71 \%$ male and $29 \%$ female students used the application. It also shows that $49 \%$ of the participants were over 27 years old, and $86 \%$ of them study Information Processing Science, while the rest study some technology related program. We can claim that all participants have high level of knowledge about computers and technology. Furthermore, the data about daily computer usage is collected, and it shows that $43 \%$ of participants use computer for 6-8 hours per day and $31 \%$ over 8 hours per day. Furthermore, it shows that they use computer 2-6 hours per day for educational purposes. We can claim that participants are active computer users with proper knowledge about technology, which is an expected characteristic in young unemployed users. Furthermore, we investigate the previous experience in gamified application, and it depicts that 28 out of 35 participants had previously engaged in applications with integrated gamification elements. Fortyseven students used the application, while only 35 participants answered the questionnaire.

\section{B. Pilot test}

Pilot test is conducted in an employment event, called MegaMatchmaking, which took place at Oulu City Theater on 14th Nov, 2017. Young unemployed participants are considered as representative of main target user group of HireMe. On the other hand, EchoMe includes wider range of target users. Users performed predefined tasks and they were encouraged to think aloud. It clarifies confusing tasks and usability flaws in the application design. Users filled pre-questionnaire and post-questionnaire during the task scenarios. The pilot test aims to improve the test process for the assessment test. The data is collected by screen recording application, notes and audio recorder. Five test subjects participated the test, and movie tickets were given as incentive to recruit them.

\section{Assessment test}

There were predefined task and activities for the user to perform in assessment test. Pre-questionnaire before the test and post-questionnaire after the task scenarios were provided by the test moderator. Demographic data and users experience level of games and gamification were collected through pre-questionnaire. Likert-scale data about user attitudes towards of EchoMe usability and the concept of involving gamification elements were collected through Post-questionnaire.

A private room in the main library of University of Oulu was booked for the tests, while two test sessions were held in BusinessOulu premises at Oulu City Centrum. Test subjects were in the age range of 25-30, which follows the requirement of HireMe users, as it is designed for unemployed young adults, while both applications (HireMe and EchoMe) were tested at the same time. Seven test subjects participated in the test, which is considered enough number for usability test by Ref. [12].

Test users age varied from 25-37 years and average age was 29, and four out of seven were female. Their education varied from goldsmith to doctor. Five users had university level education. Four participants had previously used applications that use gamification elements, and three users played games on daily basis, while the rest play less than once a month. Testing tools consisted of laptop, OBS Studio software, task list and pencil. OBS studio is open source screen recording software that was used to record test sessions. The testing Laptop was Dell Latitude 12,5" and it ran Windows 7 operating system, and EchoMe was used via Chrome internet browser.

\section{Test result}

As result of the remote test, there is no strong evidence that shows students prefer EchoMe to improve 
presentation skills than a real-life presentation practices. Most students are not willing to present all school presentations via EchoMe and only half of the users find it joyful to use. Overall, the users were not significantly satisfied with the use of EchoMe, which is mostly because of the design flaws. Nevertheless, the students thought that using EchoMe was easy. In addition, 29 out of 35 users would have preferred to see their points from the beginning of the course. Positive sign is also that most users thought that comparing points from the beginning of the course would have motivated them to use EchoMe longer. A critical aspect of the study is that most students were not comfortable sharing a video presentation on a website, and it is assumed that it can be the cause of unsatisfied responses. Considering the usability design, most students faced system error while using EchoMe application and that of course affects the overall satisfaction.

Since the pointing system is designed subjectively before the course starts, the total points that users collected during the course do not represent the actual student effort. To investigate the student involvement in the system, an activity list is formed by recording the points separately for different activities such as uploading, commenting, rating and etc. In the other word, it shows that if they became more active on the second half of the course after the leaderboard panel is revealed to them. Activities in the first 20 days were mandatory, while it was optional to use the EchoMe in the second 20 days to improve their work. We assume that in regular school work, some students tend to only participate in the mandatory work and skip the optional tasks. Interesting result demonstrated that $50 \%$ of the students were more active on the second half of the course when they knew about the leaderboard panel, and they wanted to improve their collected points in the system.

Free answer questions revealed that users were disappointed at the visual design, and some missing features like editing video titles and deleting videos, which are quite relevant features in similar applications. Many of the users complained about slow main page and video downloading, and they also considered the main page to be messy because all the videos were presented on the EchoMe page. Users considered EchoMe to be useful for sharing and improving video presentations by improving the design. They think it is a simpler way to do a presentation compared to real-life presentation. Some users complained that they did not receive any feedback from their peers, and this is significant weakness of EchoMe because the learning is based on the feedback. However increasing number of users who are involved in the application can mitigate the problem.

It was interesting to see that remote usability test revealed different problems than assessment test. One obvious factor is that remote usability test had 47 test users and assessment test had seven. The set up between the tests is also different. It is different to use the application if there are more other videos uploaded onto the server compared to a few videos. In the remote test users made their own video, which revealed problems with the video format compatibility, while in the assessment test a video was prepared for them to upload.

Most troublesome tasks were commenting a video, deleting comment and uploading a video. When commenting on a video, the comment does not appear unless the user goes back to the previous page and then return to the video page. All test users wanted to delete their comment from the same page where they submitted it. Three out of seven users asked help how to do it and others were able to find comment editor after browsing pages. Comment editor is a good idea if you have many comments on different videos but there should also be possibility to delete the comments from the video page. Slow uploading video and slow streaming video were other usability issues. Those are most likely caused by the slow server, which also seemed to be unresponsive sometimes.

We aggregated the result through mean values from Likert questionnaire in the assessment test. Test users did not have any concrete attitude towards pointing system, leaderboard panel and concept of gamified applications. Interesting fact was that points did not have any meaning for most of the test users, and they did not know how they felt about comparing points against others. Based on this, gamification element is not perceived significantly interesting to them, which differs from the findings in remote usability test, and it derived us to form hypothetical reasoning. It is important to notice that the difference between sample sizes and long using period in two test. Remote usability test had 35 participants who used the system for 40 days and assessment test only seven who used the system in one session.

In remote usability test, test subject used the EchoMe for 40 days, so they had a proper understanding of the application workflow, compared to test users of the assessment test, who used the application for approximately 15 minutes.

In the assessment test, navigation structure of the application was considered meaningful among the test users, while they believe the visual appearance should be improved.

\section{CONCLUSION}

Gamification has been used as a motivational factor to involve users in many applications in the recent years. The study aims to investigate user's attitude toward gamification elements in EchoMe application, which is a peer support tool to improve presentation skill. The evaluation phase focuses on usability of the system and gamification concept in design.

Result of the study shows that even though the users were not satisfied with the usability of the application, the gamification elements significantly enhance user's involvement in the system. Another interesting finding is that students dislike using the system with leaderboard page and visible pointing system for tasks in university and school. Therefore, a university school activity is not a proper setting to evaluate the system, and students' activities are biased by other invisible factors. As another limitation to the study, not all the test participants were exactly the representative of the application target users. Since most of the received negative feedback aims the flaws in user interface design, further evaluation should be conducted after improving the design of the application and enhancing the usability of available features.

\section{ACKNOWLEDGMENT}

This contribution is based on the results from Mika Haapaniemi master thesis work at the University of Oulu, 
Oulu Advanced Research on Service and Information Systems (OASIS) research group. The research presented in this paper was supported by Serious Games platforms for Business and Education (SEGABU) project. We thank Ilkka Hietaniemi for his contribution in the concept design.

\section{REFERENCES}

[1] Ministry of Economic Affairs and Employment (2017), http://julkaisut.valtioneuvosto.fi/bitstream/handle/10024/160692/T EM\%20tyopoliittinen\%20aikakauskirja\%201_2018.pdf, September 2017.

[2] C. Redeker, M. Leis, M. Leendertse, Y. Punie, G. Gijsbers, P.A. Kirschner, S. Stoyanov and B. Hoogveld, "The future of learning: Preparing for change," 2012.

[3] S. Huang, C. Wei, P. Yu and T. Kuo, "An empirical investigation on learners' acceptance of e-learning for public unemployment vocational training," International Journal of Innovation and Learning, vol. 3, pp. 174-185, 2005.

[4] J.P. Hausknecht, D.V. Day and S.C. Thomas, "Applicant reactions to selection procedures: An updated model and meta-analysis," Person.Psychol., vol. 57, pp. 639-683, 2004.

[5] R.A. Posthuma, F.P. Morgeson and M.A. Campion, "Beyond employment interview validity: A comprehensive narrative review of recent research and trends over time," Person.Psychol., vol. 55, pp. 1-81, 2002.

[6] A.M. Saks, "Antecedents and consequences of employee engagement," J.Manage.Psychol., vol. 21, pp. 600-619, 2006.

[7] S. Deterding, D. Dixon, R. Khaled and L. Nacke, "From game design elements to gamefulness: defining gamification," in Proceedings of the 15th international academic MindTrek conference: Envisioning future media environments, pp. 9-15, 2011 .

[8] M. Oliveira and S. Petersen, "The Choice of Serious Games and Gamification," in International Conference on Serious Games Development and Applications, pp. 213-223, 2014

[9] J. Hamari and V. Eranti, "Framework for Designing and Evaluating Game Achievements." in Digra Conference, 2011.

[10] N. Thomas, The Concise Adair on Communication and Presentation Skills, Thorogood Publishing, 2003.

[11] Z. Fitz-Walter, D. Tjondronegoro and P. Wyeth, "Orientation passport: using gamification to engage university students," in Proceedings of the 23rd Australian computer-human interaction conference, pp. 122-125, 2011.

[12] J. Rubin and D. Chisnell, Handbook of usability testing: howto plan, design, and conduct effective tests, John Wiley \& Sons, 2008. 